\title{
Nodal upstaging after thoracoscopic versus open lobectomy
}

In this nationwide database study from Denmark, nodal upstaging and survival after thoracoscopic and open lobectomy for clinical stage I non-small cell lung cancer (NSCLC) was investigated. Clinical data for all patients diagnosed with lung cancer in Denmark are obligatorily reported to the national Danish Lung Cancer Registry (DLCR). Cross-linkage with the DLCR and other national registers is used to validate completeness of data and survival status is obtained from the Centralised Civil Registry. Surgery for lung cancer in Denmark is only performed by thoracic surgeons at four university hospitals.

The DLCR was used to identify 1513 patients who underwent lobectomy for clinical stage I NSCLC between 2007 and 2011. Thoracoscopic lobectomy was performed in 47\% and thoracotomy was used in 53\%. The use of a minimally invasive approach increased gradually during the study period. Information regarding sex, age, Charlson comorbidity index, use of positron-emission tomography, the preoperative staging method, clinical TNM (cTNM), surgical approach (thoracoscopic or open), lobe resected, number of lymph node stations harvested, pathological TNM (pTNM), final histology, and survival time was collected from the DLCR. Nodal upstaging (the discovery of lymph node metastases in the surgical samples of patients clinically staged to be node negative) was identified by comparing cTNM with pTNM and occurred in 281 patients $(18.6 \%)$ and was significantly more frequent after thoracotomy $(24.6 \%$ vs $11.9 \% ; \mathrm{p}<0.001)$. Although crude estimates of survival during a median follow-up of 28 months favoured a less invasive procedure, the adjusted survival showed no difference between thoracoscopic and open surgery (HR $0.98 ; 95 \%$ CI 0.80 to 1.22 ).

By design, the study was limited by selection bias due to the non-randomised treatment allocation, that is, the operative approach was at the discretion of the surgeon, and the reason for selecting either thoracoscopic or open lobectomy was not recorded in the register. Moreover, neither the rate nor the reason for intraoperative conversion from thoracoscopic to open procedure was available from the DLCR.

Previous studies have questioned the efficacy of the surgical lymph node evaluation during minimally invasive lobectomies for NSCLC. Node evaluation is important because the findings may affect the decision for adjuvant chemotherapy. Nodal upstaging was more common after open lobectomy in this study, although survival was similar between treatment groups.

\section{Ulrik Sartipy}

Correspondence to Dr Ulrik Sartipy, Department of Cardiothoracic Surgery and Anesthesiology, Karolinska University Hospital, Stockholm SE-171 76, Sweden; Ulrik.Sartipy@karolinska.se

Competing interests None.

Provenance and peer review Not commissioned; internally peer reviewed.

To cite Sartipy U. Thorax 2014;69:353.

Published Online First 10 July 2013

Thorax 2014;69:353. doi:10.1136/thoraxjnl-2013-204085

\section{REFERENCE}

1 Licht PB, Jørgensen OD, Ladegaard L, et al. A national study of nodal upstaging after thoracoscopic versus open lobectomy for clinical stage I lung cancer. Ann Thorac Surg 2013 Published Online First: 16 April 2013. doi: 10.1016/j.athoracsur.2013.04.011 